\title{
Towards a functional hypothesis relating anti-islet cell autoimmunity to the dietary impact on microbial communities and butyrate production
}

David Endesfelder ${ }^{1}$, Marion Engel ${ }^{1}$, Austin G. Davis-Richardson², Alexandria N. Ardissone ${ }^{2}$, Peter Achenbach ${ }^{3}$, Sandra Hummel ${ }^{3}$, Christiane Winkler ${ }^{3}$, Mark Atkinson ${ }^{4}$, Desmond Schatz $^{4}$, Eric Triplett ${ }^{2}$, Anette-Gabriele Ziegler ${ }^{3}$ and Wolfgang zu Castell ${ }^{1,5^{*}}$

\begin{abstract}
Background: The development of anti-islet cell autoimmunity precedes clinical type 1 diabetes and occurs very early in life. During this early period, dietary factors strongly impact on the composition of the gut microbiome. At the same time, the gut microbiome plays a central role in the development of the infant immune system. A functional model of the association between diet, microbial communities, and the development of anti-islet cell autoimmunity can provide important new insights regarding the role of the gut microbiome in the pathogenesis of type 1 diabetes.

Results: A novel approach was developed to enable the analysis of the microbiome on an aggregation level between a single microbial taxon and classical ecological measures analyzing the whole microbial population. Microbial co-occurrence networks were estimated at age 6 months to identify candidates for functional microbial communities prior to islet autoantibody development. Stratification of children based on these communities revealed functional associations between diet, gut microbiome, and islet autoantibody development. Two communities were strongly associated with breast-feeding and solid food introduction, respectively. The third community revealed a subgroup of children that was dominated by Bacteroides abundances compared to two subgroups with low Bacteroides and increased Akkermansia abundances. The Bacteroides-dominated subgroup was characterized by early introduction of non-milk diet, increased risk for early autoantibody development, and by lower abundances of genes for the production of butyrate via co-fermentation of acetate. By combining our results with information from the literature, we provide a refined functional hypothesis for a protective role of butyrate in the pathogenesis of type 1 diabetes.

Conclusions: Based on functional traits of microbial communities estimated from co-occurrence networks, we provide evidence that alterations in the composition of mucin degrading bacteria associate with early development of anti-islet cell autoimmunity. We hypothesize that lower levels of Bacteroides in favor of increased levels of Akkermansia lead to a competitive advantage of acetogens compared to sulfate reducing bacteria, resulting in increased butyrate production via co-fermentation of acetate. This hypothesis suggests that butyrate has a protective effect on the development of anti-islet cell autoantibodies.
\end{abstract}

Keywords: Gut microbiome, Type 1 diabetes, Islet immunity, Mucin degradation, Interaction networks, Butyrate

\footnotetext{
* Correspondence: castell@helmholtz-muenchen.de

'Scientific Computing Research Unit, Helmholtz Zentrum München, Munich,

Germany

${ }^{5}$ Department of Mathematics, Technische Universität München, Munich,

Germany

Full list of author information is available at the end of the article
} 


\section{Background}

The human holobiont is defined as the human host together with all its associated microorganisms [1] colonizing various regions of the human body. By far, the largest community of microorganisms resides in the large intestine where an estimated number of $10^{12}$ bacteria per gram can be found in stool samples [2]. The microbial community of the gastrointestinal tract serves several functions including fermentation of unabsorbed nutrients, partnering the human immune system, or providing barrier against pathogenic attacks [2, 3]. Constituting an important ecosystem within the human body, gut microorganisms are increasingly considered to play a crucial role in the development of autoimmune diseases $[4,5]$ such as type 1 diabetes (T1D).

T1D is an autoimmune disease that results in destruction of insulin producing cells in the islets of Langerhans which is preceded by the development of islet autoantibodies. Thus, seroconversion to islet autoantibodies is considered to be an important checkpoint in pathogenesis of T1D [6]. Interestingly, in individuals with a high risk background for T1D, incidences of seroconversion to autoantibody positivity peak within the period of 9 months to 2 years of age and a second, less prominent peak has been observed at approximately 8 years of age [6]. Beyond this, the first months of life are a distinguished period for the development of the human immune system. Thus, major switches in the transformation of mucosal barrier function in the gut reside within this time period [7]. While it is generally agreed on that genetic background constitutes approximately $60 \%$ of T1D risk [8], the remaining propensity to disease has been attributed to several environmental factors including diet, early infections, or mode of delivery $[9,10]$. Among these, early dietary factors along with early programming of the immune system can be associated with microorganisms colonizing the body. Being the organ where nutritional components have to pass the epithelial barrier, as well as a site of high metabolic activity, the gut ecosystem has the highest density of immune cells within the human body [11]. This said, reports on T1D risk being associated with gut bacteria do not yet provide a clear picture $[5,12,13]$.

Several studies have shown an association between altered gut microbial communities and autoimmunity [14-19]. However, although several researchers analyzed the gut microbiome after autoantibody development [14, $16,19]$ or T1D onset $[20,21]$, few studies focus on the period prior to autoantibody development [15, 17, 18]. Among the first, Giongo et al. [17] reported a shift in the ratio of Bacteroidetes and Firmicutes in a group of eight Finnish children. Recently, higher abundances of Bacteroides dorei prior to autoantibody seroconversion have been associated with increased risk of islet autoantibodies [15]. This finding aligns with earlier reports on increases of abundances of Bacteroides spp. subsequent to islet autoantibodies and/or T1D disease onset $[14,20,21]$. Brown et al. [16] presented a metagenomics analysis showing lower portions of butyrate-producing and mucin-degrading bacteria in autoantibody positive children. In the German BABYDIET study, differences on the level of the single bacterium could not be seen, while the overall microbial community structure was compromised in individuals who later developed islet autoantibodies [18].

Early dietary effects, such as breast-feeding, have been reported to influence T1D development. However, contradictory results have been published. In general, breast-feeding seems to exhibit a protective role for T1D [22] in retrospective analyses, while prospective cohorts $[10,23]$ have not been able to confirm this observation. Rather, there is increasing evidence that increased risk for T1D is associated with early introduction of complex diet, in particular gluten and cereals [10, 24, 25], or fruits and berries [23, 26].

To our knowledge, the German BABYDIET cohort $[18,27]$ is currently the largest prospective cohort providing detailed dietary protocols as well as longitudinal microbial 16S rRNA amplicon sequencing data and information about the development of islet autoantibodies. Thus, this cohort provides a unique opportunity to analyze the association between infant diet and gut microbial communities with respect to the development of islet autoantibodies. Being a unique source, we reanalyzed the publicly available $16 \mathrm{~S}$ rRNA amplicon data with regard to the impact of the gut microbiome on the development of islet autoimmunity prior to seroconversion. Our aim hereby was to derive a functional hypothesis which may provide guidelines for future design of cohorts.

When addressing what key functionalities stabilize the gut microbial community and balance its interaction with the host, neither the properties on the level of the community as a whole nor the taxonomic identities provide sufficient information. Thus, our objective was to provide a level for functional analysis that reaches beyond a single microbial taxon and at the same time being finer than classical measures on the community level such as microbial diversity. Through applying community analysis on co-occurrence networks of bacteria, we show that three communities can be identified representing functional groups of microbial genera. In particular, this intermediate level of observation allows dissecting the "dietary age" of the children. The latter turned out to have much stronger influence on the gut microbial community than the actual biological age. Stratification of children on the level of microbial communities unravels alterations in the ensemble of mucin degrading bacteria in the intestinal flora of children who 
later developed islet autoantibodies. Specifically, lower abundances of Akkermansia in favor of Bacteroides, together with a functional shift in butyrate metabolism, associate with early introduction of non-milk diet, in particular meat, as well as with higher risk of seroconversion early in life. Summarizing, our analysis suggests that instead of the biological age, the dietary age should be considered for the analysis of the gut microbiome with respect to autoantibody development. Due to substantial variations regarding the biological age at seroconversion, accounting for diet is of particular importance when dealing with associations between microbial factors and seroconversion.

\section{Methods}

\section{BABYDIET study and 16S rRNA gene sequencing}

In total 298 stool samples from 44 children participating in the BABYDIET study were used for microbiome analysis. These included 147 samples from 22 children who developed persistent anti-islet cell autoantibodies at a median age of 1.54 years (IQR 0.90 years and maximum 2.45 years) and 151 samples from 22 children who remained anti-islet cell autoantibody negative. These latter subjects were also matched for date of birth. On average 6.8 stool samples per child were taken from age 0.24 to 3.2 years. To analyze microbial communities before the development of the first autoantibody, we used samples from children that had at least one probe between age 3 and 9 months. The probe closest to 6 months of age was used if several probes per child were available. This resulted in a total of 40 children, including 19 autoantibody positive and 21 autoantibody negative children. None of these children had already developed their first positive autoantibody. A detailed description of the sample collection, the questionnaire on dietary intake, and the BABYDIET cohort can be found in [18] and [27]. In brief, data on breast-feeding, the duration of breast-feeding, and the introduction of solid food (gluten-free and gluten-containing cereals, vegetables, fruits, potato, and meat) were taken from daily food records completed by the child's parents. Written informed consent was obtained from the parents. The study was approved by the ethics committee of the Ludwig-Maximilian-University, Munich, Germany (Ethikkommission der Medizinischen Fakultät der Ludwig-Maximilians Universität No. 329/00). Stool sample collection and 16S rRNA gene sequencing was performed as described in [18]. In brief, PCR was performed at an initial denaturation temperature of $94^{\circ}$ $\mathrm{C}$ for $3 \mathrm{~min}$, followed by 20 cycles of $94^{\circ} \mathrm{C}$ for $45 \mathrm{~s}, 50^{\circ}$ $\mathrm{C}$ for $30 \mathrm{~s}$, and $65^{\circ} \mathrm{C}$ for $90 \mathrm{~s}$. A final elongation step at $65{ }^{\circ} \mathrm{C}$ was run for $10 \mathrm{~min}$. PCR products were purified using the Qiagen $^{\text {TM }}$ PCR purification kit following the manufacturer's protocol [18]. The V4 region of the 16S
rRNA genes was used, and bacterial $16 \mathrm{~S}$ genes were amplified using the primers $515 \mathrm{~F}$ and 806R. Deviating from the procedure described in [18], sequences were aligned to the Greengenes 13.8 database [28] at $97 \%$ identity using the USEARCH program version 6.022 and low quality reads were trimmed as described in [15]. The Illumina $16 \mathrm{~S}$ sequences are available from NCBI's short read archive (accession number SRP063271). To account for the influence of sequencing artifacts, operational taxonomic units (OTUs) that were not present with $\geq 50$ reads in $\geq 10$ samples were excluded from all further analyses.

\section{Statistical analysis}

The aim of this study was to develop a method for the identification of stable gut microbial communities from interaction networks prior to the development of anti-islet autoantibodies, and thus, providing a novel opportunity to analyze gut microbial communities on a level beyond the single bacterium and the microbial community as a whole. In a first step, we estimated microbial communities based on co-occurrence networks. Next, microbial communities were validated by analyzing their association with dietary factors and last but not least microbial communities were tested for associations with the development of anti-islet autoantibodies. In total, 1048 OTUs were detected, and classified OTUs $(N=563)$ were aggregated on genus level using the Greengenes 13.8 database [28]. To avoid bias due to sequencing artifacts, genera with less than $0.01 \%$ abundance within the total number of reads were neglected for community analysis. The CCREPE [29] method was used to estimate Spearman's rank correlations $(\rho)$ with $P$ values corrected for compositional data. An edge was set between two bacterial genera if the $P<0.05$ and $\rho>0.4$. For further network analysis, the largest connected component of the network was selected. Community analysis was applied to the network using the Markov Dynamics clustering algorithm by [30] implemented in MATLAB ${ }^{\circ}$. This algorithm allows identification of cliquelike communities within a continuous range of a parameter (i.e., Markov time), capturing dynamic characteristics of processes on the network. To determine the number of communities, we chose a number larger than two showing longest stability with respect to Markov time (see Fig. 1a). To analyze the association of the identified gut microbial communities with diet and autoantibody development, children were stratified based on genera in each of the communities separately. Based on UniFrac distance [31], clustering of children was performed with the Partitioning Around Medoids method [32], and the number of clusters was determined by the Calinski-Harabasz method implemented in the R package $f p c$ [33]. The number of food ingredients (potato, meat, vegetables, fruit, and formula milk) was used to determine a score for the complexity of 


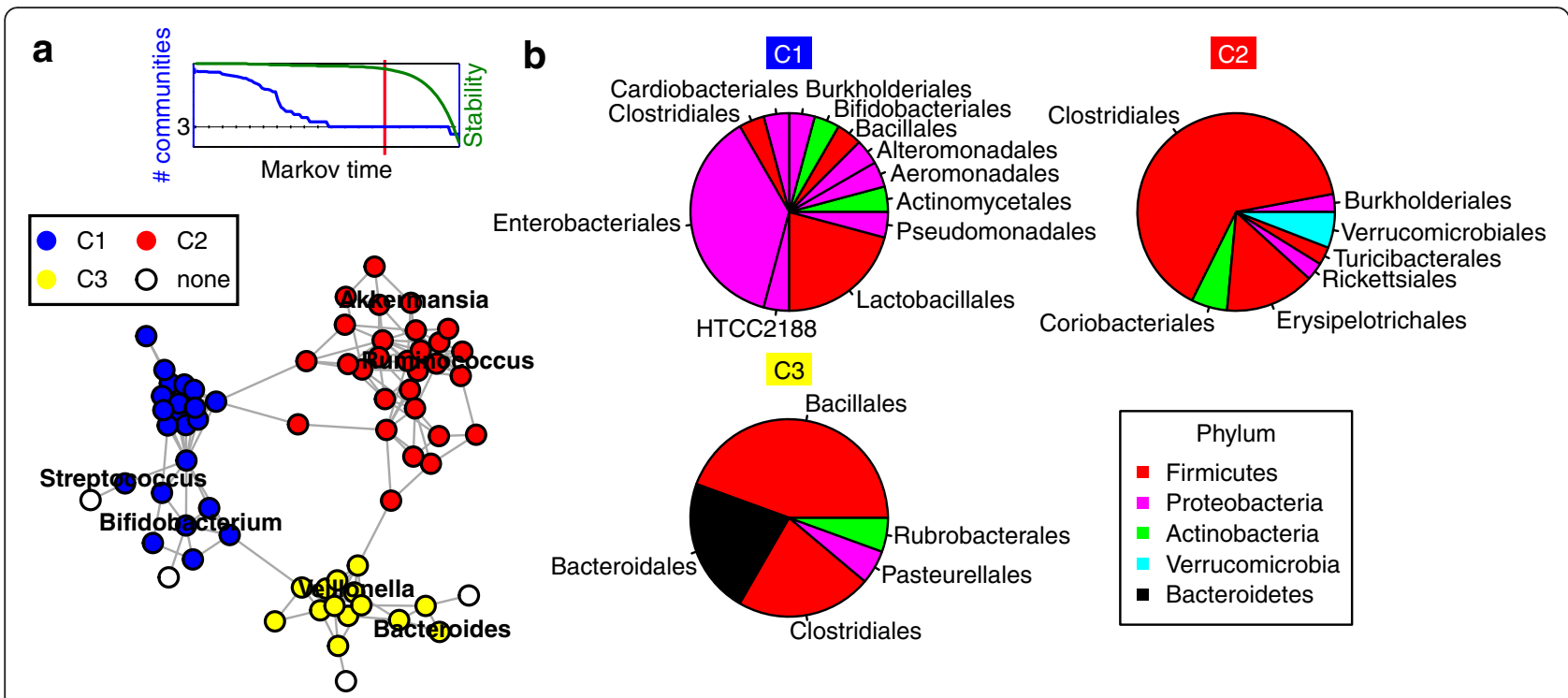

Fig. 1 Description of co-occurrence network-based microbial communities. a Co-occurrence network of microbial genera at age 6 months. Each node represents one microbial genus and colors (blue, red, yellow) indicate microbial communities. Genera that had one or no connection to other genera were not assigned to communities (white nodes) (b) Taxonomic composition of the communities C1, C2, and C3. The segments of the pies show the percentage of bacterial genera in a community that can be summarized on order level. Similarly, the colors show the percentage of bacterial genera in a community that can be summarized on phylum level

the diet for each individual. $P$ values for the differences in breast-feeding frequencies between subgroups of children were obtained by comparing the number of children who were still breast-fed vs. children who were no longer breastfed by two-sided Fisher's exact tests. Similarly, $P$ values for food complexity were obtained by comparing individuals who were already fed $>3$ vs. individuals who were fed $\leq 3$ different food ingredients by two-sided Fisher's exact tests. Finally, the PICRUSt method [34] was used to infer in silico metagenomes based on the KEGG database [35, 36] from the 16S OTU table. To compare subgroups of children regarding their genetic potential to produce butyrate via the phosphotransbutyrylase and butyrate kinase pathway (K00634 and K00929) or the butyryl CoA:acetate CoA transferase pathway (K01034 and K01035), we compared the relative abundances of the KEGG genes (KOs) required for the last steps in these pathways. All statistical analyses were performed with $R$ version 3.0.2 and MATLAB ${ }^{\circ}$ 2012b. The full documentation of all statistical analyses can be found in Additional file 1, and the required OTU and KO abundance tables can be found in Additional files 2 and 3 . If not mentioned otherwise, two-sided $P$ values were used throughout the manuscript.

\section{Results}

\section{Co-occurrence network-based bacterial communities at age 6 months}

The analysis of microbial co-occurrence patterns can provide valuable insights into factors driving the assembly of functional microbial communities. Co-occurrence networks were analyzed at 6 months of age as the focus of our approach was on the functional role of the gut microbiome with regard to the development of the infant immune system prior to autoantibody seroconversion. Furthermore, at this age, diet has a substantial impact on the microbial community as changes from breast-feeding to more complex food ingredients often occur at approximately 6 months of age. Using an a priori criterion for stability based on Markov time (see the "Methods" section), three clique-like stable communities (C1, C2, and $\mathrm{C} 3$ ) could be clearly identified in the association network of genera determined in samples of 6 months of age (Fig. 1; Additional file 4: Figure S1). Community C1 consisted to a significantly larger part of the taxonomic orders Enterobacteriales (38\%, $P<0.00001)$ and Lactobacillales $(21 \%$, $P=0.002$, Fig. $1 \mathrm{~b})$ when compared to $\mathrm{C} 2$ and $\mathrm{C} 3$ and was the only community that included genera from the order Bifidobacteriales. Community $\mathrm{C} 2$ constituted mainly of Clostridiales $(65 \%, P<0.00001)$ and Erysipelotrichales ( $15 \%, P=0.02$, Fig. $1 \mathrm{~b})$, both from the phylum Firmicutes. Thus, this group contained several specialists that are characteristic for an adult-like community, such as Ruminococcus, Blautia, or Akkermansia (Fig. 1b). Community C3 included a large proportion of Bacteroidetes (22 \%, $P=0.002$, Fig. 1b). Although Firmicutes were also providing the majority within this community $(67 \%)$, there were much less Clostridiales (22\%) present within this group compared to a significant portion of Bacillales (44 \%, $P=0.00002)$. Also 
Veillonella and some other Veillonellaceae fell within the third community. Next, we analyzed the temporal development of abundances of genera in the communities using the extended longitudinal data set (see the "Methods" section) gained from samples taken over 2 years. While the majority among dominant bacteria in communities $\mathrm{C} 1$ and $\mathrm{C} 2$ showed decreasing (C1) or increasing (C2) abundances, respectively, community $\mathrm{C} 3$ consisted of both, bacteria expressing increasing as well as decreasing abundances over time (Additional file 4: Figure S2).

Microbial communities are associated with dietary factors The associations of the gut microbial composition with breast-feeding and the introduction of solid food have been extensively described in the literature [37]. We therefore analyzed the association of diet with the three microbial communities as a validation of our approach to identify functional groups of bacteria on a community level. The taxonomic composition of communities $\mathrm{C} 1$ and $\mathrm{C} 2$ already suggested that diet might be a major factor being reflected through bacteria in these communities. In contrast, no obvious dietary pattern was seen for community $\mathrm{C} 3$, suggesting that this group characterizes additional factors influencing community composition. Stratification of children based on abundances of genera in each community (Additional file 5: Table S1) confirmed that diet associated with abundances in communities $\mathrm{C} 1$ and $\mathrm{C} 2$. While community $\mathrm{C} 1$ showed increased abundances in breast-fed individuals (G11 and G12 in Additional file 4: Figure S3B, $P=0.012)$ and decreased abundances in children who were fed a more complex diet (G13 and G14 in Additional file 4: Figure S3C, $P=0.00006$ ), community $\mathrm{C} 2$ revealed the opposite pattern with decreased abundances in breast-fed children (G21 and G23 in Additional file 4: Figure $S 4 B, P=0.011$ ) and increased abundances in children that were given a more complex diet (G22 in Additional file 4: Figure S4C, $P=0.010$ ). While children in the Bifidobacterium-dominated subgroup G11 were mostly breast-fed without being fed formula milk in addition (Additional file 4: Figure S3B and S3D), subgroup G12 had almost equal abundances in Bifidobacterium, Streptococcus, and some Proteobacteria. At the same time, this subgroup included more children that were fed formula in addition to breast milk (Additional file 4: Figure S3B and S3D). In contrast, children showing higher abundances of Akkermansia, Ruminococcus, Clostridium, and Blautia (G13 in Additional file 4: Figure S3C, S3E and G22 in Additional file 4: Figure S4C and S4E) reflect the fact that the complexity of the food taken by those individuals was clearly higher. Although stratification of children based on abundances in community C3 showed a tendency for increased food complexity in a subgroup dominated by Bacteroides (G33 in Fig. 2), no significant associations of the subgroups could be observed with breast-feeding or food complexity (Fig. 2b, c).

\section{Association of a Bacteroides-dominated community with anti-islet autoantibody development}

After successfully validating our approach for microbial community detection in terms of their functional association with infant diet, microbial communities at 6 months of age were analyzed for their association with anti-islet autoantibody development. Stratification of children based on communities $\mathrm{C} 1$ and $\mathrm{C} 2$ did not reveal associations with autoantibody development (Additional file 4: Figure S3B and S4B). Clustering of children with respect to the abundances in community C3 resulted in three clusters (Fig. 2a, Additional file 5: Table S1). While microbial ensembles derived from children in subgroup G33 were dominated by the genus Bacteroides and showed almost no abundances in Akkermansia, samples from subgroups G31 and G32 had low Bacteroides and increased Akkermansia abundances (Fig. 2e, f). In line with reported associations of increased Bacteroides abundances in children that developed anti-islet autoimmunity or T1D, subgroup G31 comprised significantly more autoantibody negative children, compared to subgroup G33 (Fig. 2b, $P=0.041$, one-sided Fisher's exact test). Strikingly, compared to both, G31 and G32, subgroup G33 showed a significantly increased risk of early autoantibody development (Fig. 3a, $P=0.021, H R=2.8$ ). The increased abundances of Bacteroides in G33 observed at age 6 months could not be observed at later time points (Fig. 3b). Similarly, Akkermansia abundances between the subgroups became more similar at later time points (Fig. 3c). Interestingly, Bacteroides showed significantly increased abundances in children that already had meat in their diet $(P=0.007$, data not shown).

To identify indications of possible functional differences of genera belonging to community C3, we compared functional traits predicted via imputed metagenome data. We observed differences in the production of butyrate via the classical buk-pathway (indicated by the presence of phosphotransbutyrylase and butyrate kinase genes; K00634 and K00929) and the potential to produce butyrate by co-fermentation of acetate (indicated by the presence of butyryl CoA:acetate CoA transferase genes; K01034 and K01035), further on referred to as but-pathway. One can note that the route via co-fermentation yields higher butyrate outcome 


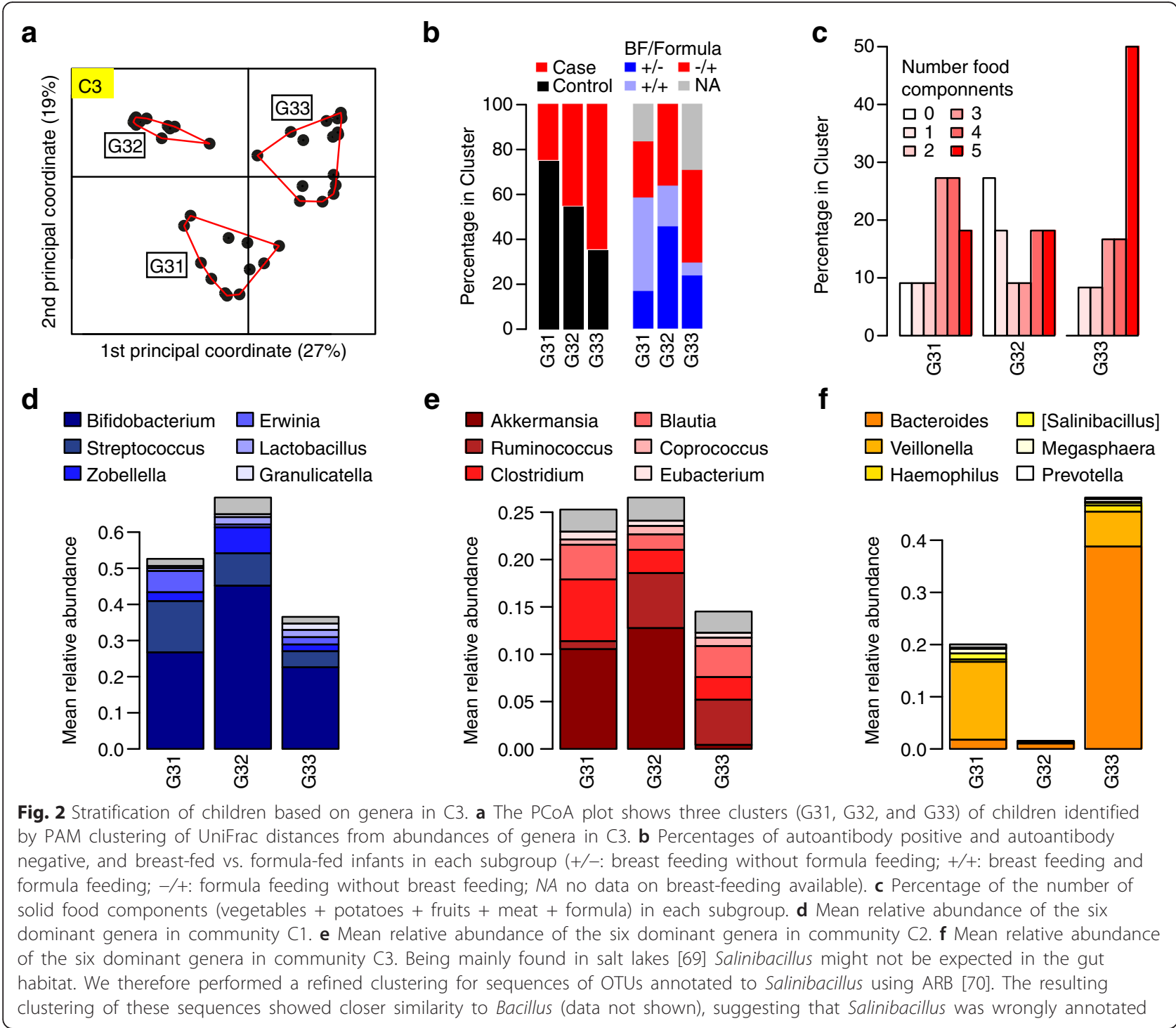

compared to the buk-pathway [38]. Interestingly, the ratio of but- vs. buk-genes was significantly lower in subgroup G33 (Fig. 3d, $P=0.00015$ ) compared to the other two communities. As a whole, clustering based on community C3 revealed a significant association of early autoantibody development in a subgroup with high Bacteroides and low Akkermansia abundances.

\section{Discussion}

The analysis of co-occurrence patterns clearly allows disentangling dietary effects on gut community composition. Children showing higher abundances in community $\mathrm{C} 1$ are still in a state of prevailing milk diet. We can identify two dietary sub-patterns through analyzing this community. There is a Bifidobacterium-dominated pattern (Additional file 4: Figure S3D, subgroup G11) that associates with higher percentages of breast- fed individuals. Moving towards an intermediate state of mixed diet including breast-milk and formula is reflected in a microbial composition which shows a more balanced community composition with lactic acid bacteria and Proteobacteria (Additional file 4: Figure S3D, subgroup G12). Likewise, community C2 comprises Clostridiaceae, Ruminococcaceae, and Lachnospiraceae, reflecting the ability of the microbial consortium to degrade an increasing variety of dietary sources, such as complex polysaccharides. This is commonly accompanied with rising abundances of genera from the phylum Firmicutes, primarily of the order Clostridiales [39]. Thus, abundances of genera belonging to community $\mathrm{C} 2$ indicate an advanced dietary age of the host (Additional file 4: Figure S4E and C, subgroup G22). Overall, communities $\mathrm{C} 1$ and $\mathrm{C} 2$ validate the assumption that the chosen community approach is a suitable way to identify 

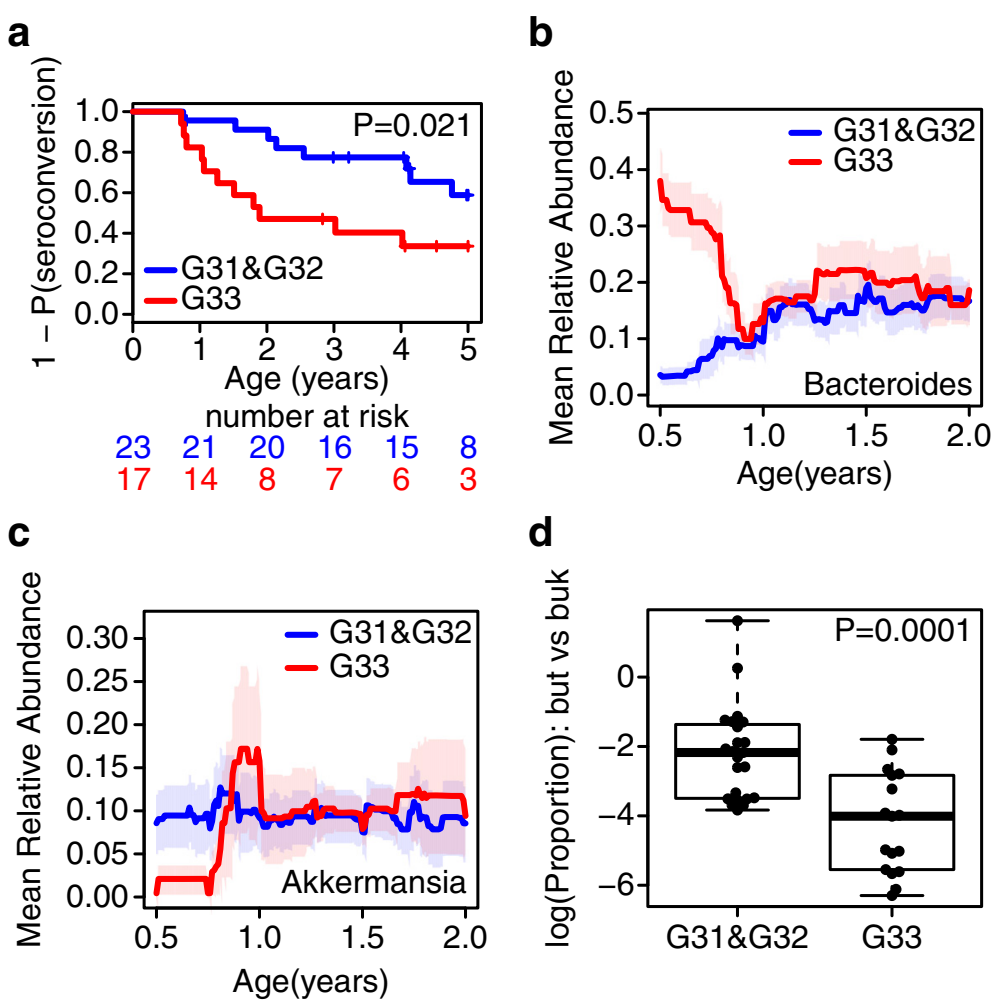

Fig. 3 Probability for autoantibody development, temporal development, and butyrate production in community C3. a Kaplan-Meier plot comparing the probability for early autoantibody development in subgroups G33 (red) vs. G31 and G32 (blue). b Bacteroides abundances vs. age in subgroups G33 (red) vs. G31 and G32 (blue). c Akkermanisa abundances vs. age in subgroups G33 (red) vs G31 and G32 (blue). d Comparison of the ratio but-genes/ buk-genes in subgroup G33 vs. subgroups G31 and G32

functional groups of bacteria, reflecting adaptation of the microbial ensembles to dynamic changes in their host-defined habitat.

Turning away from such direct dietary effects, community C3 dissects alterations with respect to the risk for developing anti-islet autoantibodies. There is a significant increase of autoantibody positive cases in subgroup G33 of children showing a community pattern with increased abundances of Bacteroides (Fig. 2f, b). Considering progression to autoimmunity, the effect becomes even more pronounced (Fig. 3a). Linking these observations to dietary patterns, the number of children exposed to complex diet (i.e., with five food components) is higher in subgroup G33 compared to G31 and G32 (Fig. 2c). Additionally, Bacteroides abundances were significantly increased in children that had meat in their diet, an observation which has also been made in another study [40]. Overall, we support observations made in the prospective cohorts, that early introduction of higher food complexity increases the risk for autoimmunity [23-25, 41]. Asking what compensates for lower abundances of Bacteroides in subgroups G31 and G32, it is striking that both subgroups have higher levels of Akkermansia (Fig. 2e). Akkermansia muciniphila is characterized by its potential to grow on mucin [42] Within this habitat, Akkermansia markedly represents a specialist with a genome containing a high number of enzymes for degradation of human-derived mucins [43]. Unlike Bacteroides species, which are also able to utilize mucins, Akkermansia cannot switch to carbohydrate fermentation derived from luminal content $[42,44]$. Generally, higher abundances of Akkermansia have been associated with a healthy gut community in several studies $[45,46]$. Most mucin degraders such as Clostridium, Bifidobacterium, and Bacteroides are not able to fully degrade mucins [47]. Therefore, mucin-degrading bacteria are frequently associated with sulfate-reducing bacteria (SRB), which use sulfate for gain of energy, thereby releasing sulfide [47]. Indeed, mucin fermentation in the colon has been demonstrated to increase the amount of released sulfate [47]. In contrast, Akkermansia are able to fully degrade mucins and its genetic content enables assimilatory utilization of sulfate [48]. Functionally, SRB as well as methanogens compete with acetogens such as Blautia and Ruminococcus for $\mathrm{H}_{2}$ produced during carbohydrate fermentation. Since hydrogen inhibits further production of short chain fatty acids (SCFAs) [39], the hydrogen gradient is a sensible driver for SCFA 
balance. In the presence of sulfate, SRB would typically outperform methanogens and acetogens in $\mathrm{H}_{2}$ utilization [49]. On the other side, $\mathrm{H}_{2}$ utilization by acetogens raises levels of acetate which then fosters higher efficiency in butyrate production via the but-pathway [38]. Butyrate has several health promoting effects. First of all, it is the major energy source for intestinal epithelial cells $[50,51]$. Consequently, butyrate has been shown to increase mucus production [52, 53]. Indeed, we observed that in contrast to children in the Bacteroides-dominated subgroup (G33), children in the Akkermansia-dominated subgroups (G31 and G32) harbor a microbiome that seems to prefer butyrate-production through cofermentation of acetate (Fig. 3d). Thus, our data leads to the hypothesis (Fig. 4) that alterations in the ensemble of mucin degraders, Bacteroides and Akkermansia, directly affect competition among hydrogenotrophic bacteria, which indirectly leaves an impact on butyrate production. Due to decreased available sulfate levels in Akkermansia-dominated communities, acetogens might have an indirect advantage which then leads to a butyrogenic effect via the but-pathway. Closing the argument towards an increased risk for anti-islet autoimmunity, there are several hypotheses currently discussed. Lower butyrate availability might impair gut integrity, thus allowing larger molecules to penetrate the epithelial barrier (leaky gut hypothesis). This line of thought frames within the context of the perfect storm hypothesis and hygiene hypothesis $[12,13]$. On the other hand, butyrate might also directly modulate immune function, in particular inflammation [50]. A third option lies in the capability of co-evolved gut inhabitants, in particular

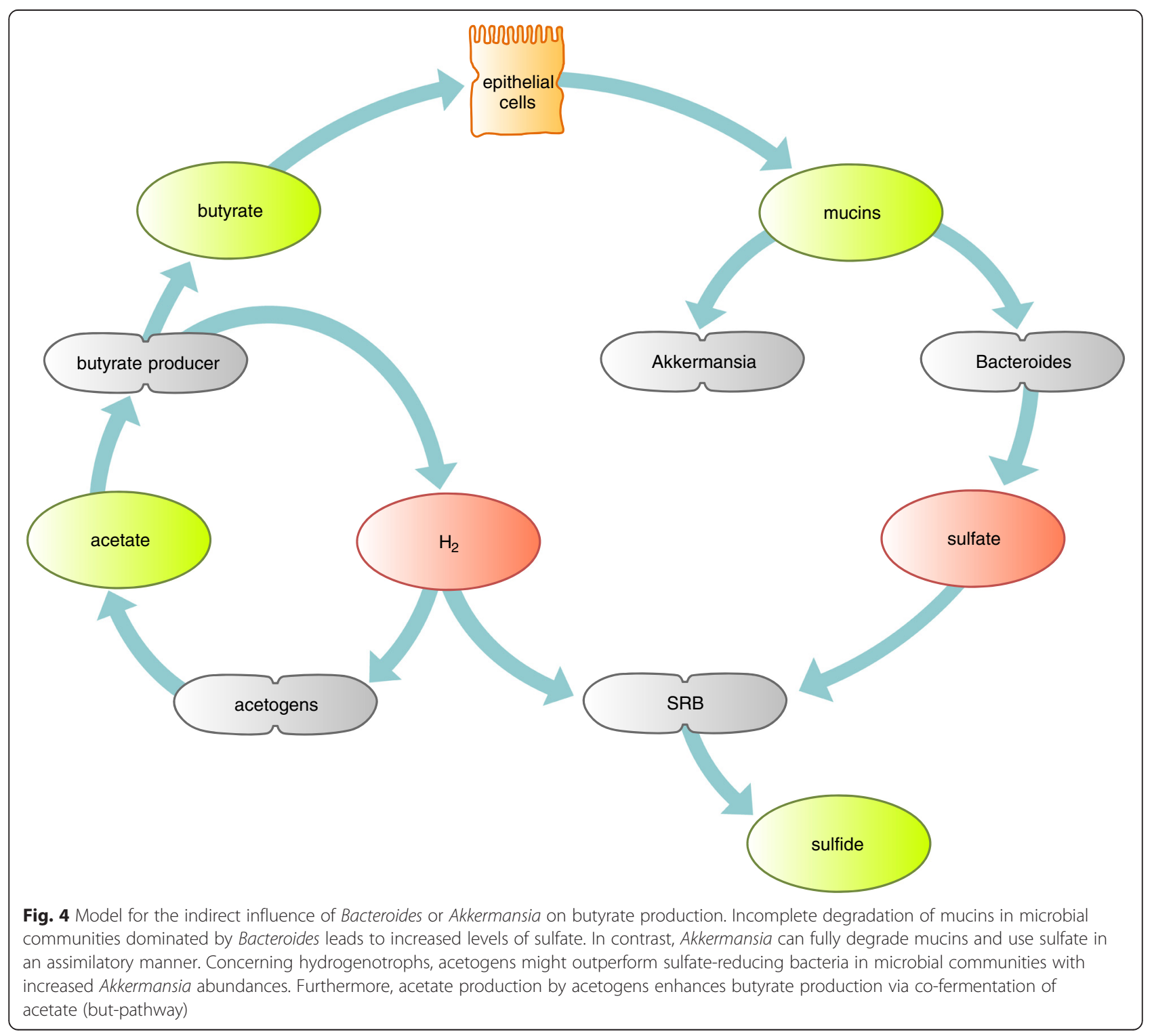


Bacteroides spp. to trigger immune signals of the host in order to defend their ecological niche [54].

Considering the host, dietary patterns clearly impact the ecological balance just discussed. Breast-feeding, for example, prepares the gut ecosystem for later colonization with mucin-degrading specialists [55], since human milk oligosaccharides (HMO) are similar in protein structure to mucins produced by gut epithelial cells [56]. It therefore seems likely that through an early introduction of complex carbohydrates, the ecological advantage for Akkermansia provided by HMO might be compromised, in favor of other mucin degraders, which can also feed on alternative substrates as, e.g., resistant starch. Being the dominant end-product of lactate fermentation in the human intestinal communities [57], acetate provides a main source of butyrate production $[58,59]$. Note that it was shown that milk-dominated diets support the butyrogenic effect of co-colonization of Bifidobacterium with acetate utilizers [60].

In silico analysis of $16 \mathrm{~S}$ rRNA gene fragments derived from SRBs revealed a high number of unclassified sequences at genus level within the different groups of sulfate reducers deposited in the databases (data not shown). Thus, the majority of SRB could not be resolved on genus level due to under-representation in OTU databases. Thus, we can only provide indirect support concerning the role of sulfate in shaping the hydrogenotrophic community. A diet rich in meat has been shown to increase exogenous sulfide in feces [61]. Thus, our observation of an association between meat consumption and increased abundance of Bacteroides is in line with the part of the hypothesis claiming that increased abundances of Bacteroides may come along with higher levels of sulfate being available in the system, thereby shifting the hydrogenotrophic community towards sulfide-releasing SRBs. Note that the presence of $B$. thetaiotaomicron had significant impact on the growth of SRB in an animal study [62].

The observation of increased abundances of Bacteroides preceding anti-islet autoimmunity shows striking parallels with other autoimmune diseases, in particular Celiac disease (CD) [63]. De Palma et al. [64] associated an HLA-DQ2 genotype being associated with both, increased risk for CD and T1D with higher abundances in the Bacteroides-Prevotella group in stool samples of children of less than 1 month of age. Of interest for our work is the fact that individuals at higher risk have been reported to harbor significantly higher abundances of SRB [64]. Sánchez et al. [63] analyzed the diversity of Bacteroides spp. in greater detail showing that infants with high HLA risk for CD showed higher prevalence of Bacteroides vulgatus. Note that in samples from adults, Leitch et al. [65] found B. vulgatus to be the only Bacteroides species detected growing on mucin. Davis-
Richardson et al. [15] also relate increased abundances of $B$. dorei, a close relative of $B$. vulgatus, with increased risk of islet-autoimmunity in the Finnish DIPP cohort. Along with observations made in the Finnish cohort [15], it seems likely that the period determining possible effects of the microbial community on pathogenesis of T1D falls within the first year of life. As can be seen from temporal data, alterations in abundances of Akkermansia fade within the second year of life (Fig. 3c). The same holds true for Bacteroides (Fig. 3b). It also appears likely that increased abundances of Bacteroides in G31 within the first year of life are due to other representatives of the genus than the abundances showing up later at the age of 2 years (Fig. 3b). A characteristic property of the genus Bacteroides, following from their long co-evolution with the human host, is the ability to switch from carbohydrate fermentation to digestion of endogenously-derived mucins [66]. Thus, depending on the availability of glycan sources in the lumen, Bacteroides community composition is expected to vary.

Overall, the combination of our results with an extensive literature search leads to a refined functional hypothesis explaining a possible role of the gut microbial community in pathogenesis of anti-islet autoimmunity. In summary, these observations support the hypothesis that increased availability of butyrate in the intestinal tract has a protective effect on development of autoimmunity and T1D. Concerning the risk of developing anti-islet autoantibodies and/or T1D several studies associated an increased risk with early introduction of complex food [10, 23-25] while protective effects of breast-feeding are discussed controversially $[10,22,23]$. The influence of diet, in particular breast-feeding on microbial composition in the large intestine is well documented. Basically, breastfeeding as well as formula-feeding seems to associate with higher abundances of lactic acid bacteria and Bifidobacterium spp. [67, 68]. Nevertheless, the overall net-effect with respect to the development of disease in our hypothesis depends on cross-feeding effects between mucin degraders, hydrogenotrophs, and butyrate producers. Thus, breast-feeding alone might or might not alter the risk, depending on the composition of the microbial ensemble. With the effect of diet on the gut microbial community being profound, evaluations of the role of the microbiome in host-microbial homeostasis and in particular its association with autoimmunity should indispensably take the nutritional habits of the subject, i.e., the dietary age into account. This conclusion is of peculiar importance for the period between birth and 3 years of age, where dramatic changes in diet leave their trace on the developing microbial ecosystem. The proposed approach using community detection in 
association networks of bacteria provides a basis for analyzing such effects on a level beyond the single bacterium and the whole community.

\section{Conclusions}

Based on a novel approach for the identification of microbial communities from co-occurrence networks, we provide a functional model for the association of gut microbial communities and the development of early anti-islet cell autoantibodies. Compared to Akkermansia-dominated subgroups of children, Bacteroides-dominated subgroups were associated with early autoantibody development and decreased potential of butyrate production via the cofermentation of acetate. The data suggest that differences in mucin degradation capabilities between the generalist $\mathrm{Bac}$ teroides and the specialist Akkermansia may lead to shifts in the abundances of acetogens vs. sulfate-reducing bacteria. Due to incomplete degradation of mucins in Bacteroides-dominated communities, sulfate-reducing bacteria might have a competitive advantage compared to acetogens regarding the removal of hydrogen. In contrast, in Akkermansia-dominated communities, abundance of acetogens might be increased due to lower levels of sulfate, leading to increased levels of butyrate via the co-fermentation of acetate. Additionally, early introduction of solid food components, such as meat can provide Bacteroides with a competitive advantage as Bacteroides is able to switch from endogenous to exogenous nutrient sources. Thus, we provide evidence that butyrate has a protective effect on the development of anti-islet cell autoimmunity and that this effect is associated with differences in composition of mucin-degrading bacteria and the early introduction of complex food. Our model provides a first step into the direction of a functional understanding of the role of the gut microbiome in the pathogenesis of type 1 diabetes. However, this model needs to be validated in larger sample size cohorts including metagenomics data.

\section{Ethics approval and consent to participate}

The study was approved by the ethics committee of the Ludwig-Maximilian-University, Munich, Germany (Ethikkommission der Medizinischen Fakultät der LudwigMaximilians Universität No. 329/00).

\section{Consent to publish}

Written informed consent was obtained from the parents.

\section{Availability of supporting data}

The 16S sequences supporting the results of this article is available in NCBI's short read archive (SRP063271; http://www.ncbi.nlm.nih.gov/Traces/study/ ?acc=SRP063271).

\section{Additional files}

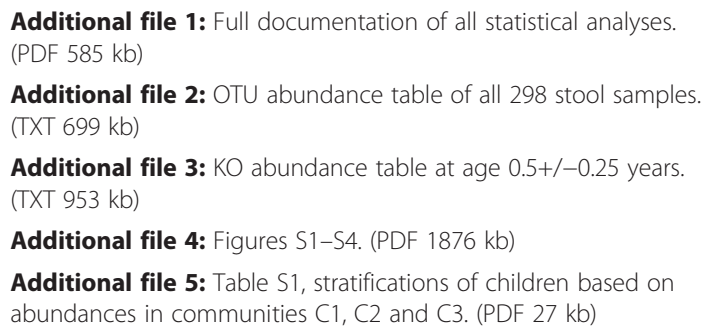

Additional file 2: OTU abundance table of all 298 stool samples. (TXT 699 kb)

Additional file 3: $\mathrm{KO}$ abundance table at age $0.5+/-0.25$ years. (TXT 953 kb)

Additional file 4: Figures S1-S4. (PDF $1876 \mathrm{~kb}$ )

Additional file 5: Table S1, stratifications of children based on abundances in communities C1, C2 and C3. (PDF 27 kb)

\section{Abbreviations}

buk: phosphotransbutyrylase and butyrate kinase; but: butyryl CoA:acetate CoA transferase; CD: celiac disease; HLA: human leukocyte antigen; HMO: human milk oligosaccharide; IQR: interquartile range; OTU: operational taxonomic unit; PCOA: principal coordinate analysis; rRNA: ribosomal ribonucleic acid; SCFA: short chain fatty acid; SRB: sulfate reducing bacteria; T1D: type 1 diabetes.

\section{Competing interests}

The authors declare that they have no competing interests.

\section{Authors' contributions}

$D E, M E$, and WZC performed data analysis and drafted the manuscript. $\mathrm{PA}, \mathrm{SH}$, and CW contributed to data collection, islet autoantibody measurement, and data analysis. AD-R, ANN, and ET performed 165 sequencing and data analysis. DS and MA contributed to data analysis, data interpretation, and manuscript writing. A-GZ is principal investigator of BABYDIET study and contributed to data collection, analysis, and manuscript writing. All authors critically reviewed and approved the manuscript.

\section{Acknowledgements}

This work was supported by grants from the Juvenile Diabetes Research Foundation (17-2012-16 and 17-2011-266), Deutsche Forschungsgemeinschaft (DFG Zl-310/14-1 to -4) and the foundation "Children With Type 1 Diabetes" (Stiftung Das Zuckerkranke Kind). The authors thank Annette Knopff (Helmholtz Zentrum München) and Melanie Bunk (Helmholtz Zentrum München) for data management and clinical assistance. No conflicts of interest have to be disclosed.

\section{Author details}

${ }^{1}$ Scientific Computing Research Unit, Helmholtz Zentrum München, Munich, Germany. ${ }^{2}$ Department of Microbiology and Cell Science, Institute of Food and Agricultural Sciences, University of Florida, Munich, USA. ${ }^{3}$ Institute of Diabetes Research, Helmholtz Zentrum München, and Forschergruppe Diabetes, Klinikum rechts der Isar, Technische Universität München, Munich, Germany. ${ }^{4}$ Department of Pediatrics, University of Florida, Gainesville, FL, USA. ${ }^{5}$ Department of Mathematics, Technische Universität München, Munich, Germany.

Received: 2 December 2015 Accepted: 22 March 2016 Published online: 26 April 2016

\section{References}

1. McFall-Ngai M, Hadfield MG, Bosch TC, Carey HV, Domazet-Loso T, Douglas AE, Dubilier N, Eberl G, Fukami T, Gilbert SF, Hentschel U, King N, Kjelleberg S, Knoll AH, Kremer N, Mazmanian SK, Metcalf $J$, Nealson K, Pierce NE, Rawls JF, Reid A, Ruby EG, Rumpho M, Sanders JG, Tautz D, Wernegreen JJ. Animals in a bacterial world, a new imperative for the life sciences. Proc Natl Acad Sci U S A. 2013:110(9):3229-36.

2. Kovatcheva-Datchary P, Arora T. Nutrition, the gut microbiome and the metabolic syndrome. Best Pract Res Clin Gastroenterol. 2013;27(1):59-72.

3. Backhed F, Ley RE, Sonnenburg JL, Peterson DA, Gordon Jl. Host-bacterial mutualism in the human intestine. Science. 2005;307(5717):1915-20.

4. Okada H, Kuhn C, Feillet H, Bach JF. The 'hygiene hypothesis' for autoimmune and allergic diseases: an update. Clin Exp Immunol. 2010; 160(1):1-9. 
5. McLean MH, Dieguez Jr D, Miller LM, Young HA. Does the microbiota play a role in the pathogenesis of autoimmune diseases? Gut. 2015;64(2):332-41.

6. Ziegler AG, Bonifacio E, Group B-BS. Age-related islet autoantibody incidence in offspring of patients with type 1 diabetes. Diabetologia. 2012; 55(7):1937-43.

7. Midtvedt AC, Carlstedt-Duke B, Midtvedt T. Establishment of a mucindegrading intestinal microflora during the first two years of human life. J Pediatr Gastroenterol Nutr. 1994;18(3):321-6.

8. Eringsmark Regnell S, Lernmark A. The environment and the origins of islet autoimmunity and type 1 diabetes. Diabet Med. 2013;30(2):155-60.

9. Knip M, Simell O. Environmental triggers of type 1 diabetes. Cold Spring Harb Perspect Med. 2012;2(7):a007690.

10. Ziegler AG, Schmid S, Huber D, Hummel M, Bonifacio E. Early infant feeding and risk of developing type 1 diabetes-associated autoantibodies. JAMA. 2003;290(13):1721-8

11. Mowat AM, Agace WW. Regional specialization within the intestinal immune system. Nat Rev Immunol. 2014;14(10):667-85.

12. Atkinson MA, Chervonsky A. Does the gut microbiota have a role in type diabetes? Early evidence from humans and animal models of the disease. Diabetologia. 2012;55(11):2868-77.

13. Dunne JL, Triplett EW, Gevers D, Xavier R, Insel R, Danska J, Atkinson MA. The intestinal microbiome in type 1 diabetes. Clin Exp Immunol. 2014;177(1):30-7.

14. de Goffau MC, Luopajarvi K, Knip M, Ilonen J, Ruohtula T, Harkonen T, Orivuori L, Hakala S, Welling GW, Harmsen HJ, Vaarala O. Fecal microbiota composition differs between children with beta-cell autoimmunity and those without. Diabetes. 2013;62(4):1238-44.

15. Davis-Richardson AG, Ardissone AN, Dias R, Simell V, Leonard MT, Kemppainen KM, Drew JC, Schatz D, Atkinson MA, Kolaczkowski B, llonen J, Knip M, Toppari J, Nurminen N, Hyoty H, Veijola R, Simell T, Mykkanen J, Simell O, Triplett EW. Bacteroides dorei dominates gut microbiome prior to autoimmunity in Finnish children at high risk for type 1 diabetes. Front Microbiol. 2014;5:678.

16. Brown CT, Davis-Richardson AG, Giongo A, Gano KA, Crabb DB, Mukherjee N, Casella G, Drew JC, Ilonen J, Knip M, Hyoty H, Veijola R, Simell T, Simell O, Neu J, Wasserfall CH, Schatz D, Atkinson MA, Triplett EW. Gut microbiome metagenomics analysis suggests a functional model for the development of autoimmunity for type 1 diabetes. PLoS One. 2011;6(10):e25792.

17. Giongo A, Gano KA, Crabb DB, Mukherjee N, Novelo LL, Casella G, Drew JC, Ilonen J, Knip M, Hyoty H, Veijola R, Simell T, Simell O, Neu J, Wasserfall CH, Schatz D, Atkinson MA, Triplett EW. Toward defining the autoimmune microbiome for type 1 diabetes. ISME J. 2011;5(1):82-91.

18. Endesfelder D, zu Castell W, Ardissone A, Davis-Richardson AG, Achenbach $P$, Hagen M, Pflueger M, Gano KA, Fagen JR, Drew JC, Brown CT, Kolaczkowski B, Atkinson M, Schatz D, Bonifacio E, Triplett EW, Ziegler AG. Compromised gut microbiota networks in children with anti-islet cell autoimmunity. Diabetes. 2014;63(6):2006-14.

19. Kostic AD, Gevers D, Siljander $H$, Vatanen $T$, Hyotylainen $T$, Hamalainen AM, Peet A, Tillmann V, Poho P, Mattila I, Lahdesmaki H, Franzosa EA, Vaarala O, de Goffau M, Harmsen $H$, Ilonen J, Virtanen SM, Clish CB, Oresic M, Huttenhower C, Knip M, Group DS, Xavier RJ. The dynamics of the human infant gut microbiome in development and in progression toward type 1 diabetes. Cell Host Microbe. 2015;17(2):260-73.

20. Murri M, Leiva I, Gomez-Zumaquero JM, Tinahones FJ, Cardona F, Soriguer F, Queipo-Ortuno MI. Gut microbiota in children with type 1 diabetes differs from that in healthy children: a case-control study. BMC Med. 2013:11:46.

21. Mejia-Leon ME, Petrosino JF, Ajami NJ, Dominguez-Bello MG, de la Barca AM. Fecal microbiota imbalance in Mexican children with type 1 diabetes. Sci Rep. 2014;4:3814.

22. Cardwell CR, Stene LC, Ludvigsson J, Rosenbauer J, Cinek O, Svensson J, Perez-Bravo F, Memon A, Gimeno SG, Wadsworth EJ, Strotmeyer ES, Goldacre MJ, Radon K, Chuang LM, Parslow RC, Chetwynd A, Karavanaki K, Brigis G, Pozzilli P, Urbonaite B, Schober E, Devoti G, Sipetic S, Joner G, lonescu-Tirgoviste C, de Beaufort CE, Harrild K, Benson V, Savilahti E, Ponsonby AL, Salem M, Rabiei S, Patterson CC. Breast-feeding and childhood-onset type 1 diabetes: a pooled analysis of individual participant data from 43 observational studies. Diabetes Care. 2012;35(11):2215-25.

23. Virtanen SM, Kenward MG, Erkkola M, Kautiainen S, Kronberg-Kippila C, Hakulinen T, Ahonen S, Uusitalo L, Niinisto S, Veijola R, Simell O, llonen J, Knip M. Age at introduction of new foods and advanced beta cell autoimmunity in young children with HLA-conferred susceptibility to type 1 diabetes. Diabetologia. 2006;49(7):1512-21.
24. Norris JM, Barriga K, Klingensmith G, Hoffman M, Eisenbarth GS, Erlich HA, Rewers M. Timing of initial cereal exposure in infancy and risk of islet autoimmunity. JAMA. 2003;290(13):1713-20.

25. Chmiel R, Beyerlein A, Knopff A, Hummel S, Ziegler AG, Winkler C. Early infant feeding and risk of developing islet autoimmunity and type 1 diabetes. Acta Diabetol. 2014;52(3):621-4.

26. Knip M, Virtanen SM, Becker D, Dupre J, Krischer JP, Akerblom HK, Group TS. Early feeding and risk of type 1 diabetes: experiences from the Trial to Reduce Insulin-dependent diabetes mellitus in the Genetically at Risk (TRIGR). Am J Clin Nutr. 2011;94(6 Suppl):1814S-20.

27. Hummel S, Pfluger M, Hummel M, Bonifacio E, Ziegler AG. Primary dietary intervention study to reduce the risk of islet autoimmunity in children at increased risk for type 1 diabetes: the BABYDIET study. Diabetes Care. 2011; 34(6):1301-5.

28. McDonald D, Price MN, Goodrich J, Nawrocki EP, DeSantis TZ, Probst A, Andersen GL, Knight R, Hugenholtz P. An improved Greengenes taxonomy with explicit ranks for ecological and evolutionary analyses of bacteria and archaea. ISME J. 2012;6(3):610-8.

29. Faust K, Sathirapongsasuti JF, Izard J, Segata N, Gevers D, Raes J, Huttenhower C. Microbial co-occurrence relationships in the human microbiome. PLoS Comput Biol. 2012;8(7):e1002606.

30. Schaub MT, Delvenne JC, Yaliraki SN, Barahona M. Markov dynamics as a zooming lens for multiscale community detection: non clique-like communities and the field-of-view limit. Plos One. 2012;7(2):e32210.

31. Lozupone C, Lladser ME, Knights D, Stombaugh J, Knight R. UniFrac: an effective distance metric for microbial community comparison. ISME J. 2011; 5(2):169-72.

32. Kaufman $L$ and PJ Rousseeuw. Clustering by means of Medoids. Statistical data analysis based on the L1-norm and related methods, edited by $Y$. Dodge, North-Holland, 1987: p. 405-416.

33. Hennig C. fpc: Flexible procedures for clustering. http://CRAN.R-project.org/ package $=f p c, 2014(R$ package version 2.1-9).

34. Langille MG, Zaneveld J, Caporaso JG, McDonald D, Knights D, Reyes JA, Clemente JC, Burkepile DE, Vega Thurber RL, Knight R, Beiko RG, Huttenhower C. Predictive functional profiling of microbial communities using 165 rRNA marker gene sequences. Nat Biotechnol. 2013;31(9):814-21.

35. Kanehisa M, Goto S. KEGG: kyoto encyclopedia of genes and genomes. Nucleic Acids Res. 2000;28(1):27-30.

36. Kanehisa M, Goto S, Sato Y, Kawashima M, Furumichi M, Tanabe M. Data, information, knowledge and principle: back to metabolism in KEGG. Nucleic Acids Res. 2014;42(Database issue):D199-205.

37. Voreades N, Kozil A, Weir TL. Diet and the development of the human intestinal microbiome. Front Microbiol. 2014:5:494.

38. Louis P, Flint HJ. Diversity, metabolism and microbial ecology of butyrateproducing bacteria from the human large intestine. FEMS Microbiol Lett. 2009;294(1):1-8.

39. Chassard C, Lacroix C. Carbohydrates and the human gut microbiota. Curr Opin Clin Nutr Metab Care. 2013;16(4):453-60

40. Wu GD, Chen J, Hoffmann C, Bittinger K, Chen YY, Keilbaugh SA, Bewtra M, Knights D, Walters WA, Knight R, Sinha R, Gilroy E, Gupta K, Baldassano R, Nessel L, Li H, Bushman FD, Lewis JD. Linking long-term dietary patterns with gut microbial enterotypes. Science. 2011;334(6052):105-8.

41. Knip M, Virtanen SM, Akerblom HK. Infant feeding and the risk of type 1 diabetes. Am J Clin Nutr. 2010;91(5):1506S-13.

42. Derrien $M$, Vaughan EE, Plugge CM, de Vos WM. Akkermansia muciniphila gen. nov., sp. nov., a human intestinal mucin-degrading bacterium. Int J Syst Evol Microbiol. 2004;54(Pt 5):1469-76.

43. van Passel MW, Kant R, Zoetendal EG, Plugge CM, Derrien M, Malfatti SA, Chain PS, Woyke T, Palva A, de Vos WM, Smidt H. The genome of Akkermansia muciniphila, a dedicated intestinal mucin degrader, and its use in exploring intestinal metagenomes. PLoS One. 2011;6(3):e16876.

44. Ouwerkerk JP, de Vos WM, Belzer C. Glycobiome: bacteria and mucus at the epithelial interface. Best Pract Res Clin Gastroenterol. 2013;27(1):25-38.

45. Png CW, Linden SK, Gilshenan KS, Zoetendal EG, McSweeney CS, Sly LI, McGuckin MA, Florin TH. Mucolytic bacteria with increased prevalence in IBD mucosa augment in vitro utilization of mucin by other bacteria. Am J Gastroenterol. 2010;105(11):2420-8.

46. Swidsinski A, Dorffel Y, Loening-Baucke V, Theissig F, Ruckert JC, Ismail M, Rau WA, Gaschler D, Weizenegger M, Kuhn S, Schilling J, Dorffel W. Acute appendicitis is characterised by local invasion with Fusobacterium nucleatum/necrophorum. Gut. 2011;60(1):34-40. 
47. Willis $\mathrm{CL}$, Cummings $\mathrm{JH}$, Neale $\mathrm{G}$, Gibson $\mathrm{GR}$. In vitro effects of mucin fermentation on the growth of human colonic sulphate-reducing bacteria. Anaerobe. 1996;2(2):117-22.

48. KEGG Database, http://www.genome.jp/kegg-bin/show_pathway? amu00920. Accessed 26 Nov 2015.

49. Dar SA, Kleerebezem R, Stams AJ, Kuenen JG, Muyzer G. Competition and coexistence of sulfate-reducing bacteria, acetogens and methanogens in a lab-scale anaerobic bioreactor as affected by changing substrate to sulfate ratio. Appl Microbiol Biotechnol. 2008;78(6):1045-55.

50. Scheppach W, Weiler F. The butyrate story: old wine in new bottles? Curr Opin Clin Nutr Metab Care. 2004;7(5):563-7.

51. Hamer HM, Jonkers D, Venema K, Vanhoutvin S, Troost FJ, Brummer RJ. Review article: the role of butyrate on colonic function. Aliment Pharmacol Ther. 2008;27(2):104-19.

52. Finnie IA, Dwarakanath AD, Taylor BA, Rhodes JM. Colonic mucin synthesis is increased by sodium butyrate. Gut. 1995;36(1):93-9.

53. Shimotoyodome A, Meguro S, Hase T, Tokimitsu I, Sakata T. Short chain fatty acids but not lactate or succinate stimulate mucus release in the rat colon. Comp Biochem Physiol A Mol Integr Physiol. 2000;125(4):525-31.

54. Buffie CG, Pamer EG. Microbiota-mediated colonization resistance against intestinal pathogens. Nat Rev Immunol. 2013;13(11):790-801.

55. Bode L. Human milk oligosaccharides: prebiotics and beyond. Nutr Rev. 2009:67 Suppl 2:S183-91.

56. Marcobal A, Southwick AM, Earle KA, Sonnenburg JL. A refined palate: bacterial consumption of host glycans in the gut. Glycobiology. 2013;23(9): 1038-46.

57. Bourriaud C, Robins RJ, Martin L, Kozlowski F, Tenailleau E, Cherbut C, Michel C. Lactate is mainly fermented to butyrate by human intestinal microfloras but inter-individual variation is evident. J Appl Microbiol. 2005: 99(1):201-12.

58. Duncan SH, Barcenilla A, Stewart CS, Pryde SE, Flint HJ. Acetate utilization and butyryl coenzyme A (CoA):acetate-CoA transferase in butyrate-producing bacteria from the human large intestine. Appl Environ Microbiol. 2002;68(10): 5186-90.

59. Duncan SH, Holtrop G, Lobley GE, Calder AG, Stewart CS, Flint HJ. Contribution of acetate to butyrate formation by human faecal bacteria. Br J Nutr. 2004;91(6):915-23.

60. De Vuyst L, Leroy F. Cross-feeding between bifidobacteria and butyrateproducing colon bacteria explains bifdobacterial competitiveness, butyrate production, and gas production. Int J Food Microbiol. 2011;149(1):73-80.

61. Magee EA, Richardson CJ, Hughes R, Cummings JH. Contribution of dietary protein to sulfide production in the large intestine: an in vitro and a controlled feeding study in humans. Am J Clin Nutr. 2000;72(6):1488-94.

62. Rey FE, Gonzalez MD, Cheng J, Wu M, Ahern PP, Gordon Jl. Metabolic niche of a prominent sulfate-reducing human gut bacterium. Proc Natl Acad Sci U S A. 2013;110(33):13582-7.

63. Sanchez E, De Palma G, Capilla A, Nova E, Pozo T, Castillejo G, Varea V, Marcos A, Garrote JA, Polanco I, Lopez A, Ribes-Koninckx C, Garcia-Novo MD, Calvo C, Ortigosa L, Palau F, Sanz Y. Influence of environmental and genetic factors linked to celiac disease risk on infant gut colonization by Bacteroides species. Appl Environ Microbiol. 2011;77(15):5316-23.

64. De Palma G, Capilla A, Nadal I, Nova E, Pozo T, Varea V, Polanco I, Castillejo G, Lopez A, Garrote JA, Calvo C, Garcia-Novo MD, Cilleruelo ML, RibesKoninckx C, Palau F, Sanz Y. Interplay between human leukocyte antigen genes and the microbial colonization process of the newborn intestine. Curr Issues Mol Biol. 2010;12(1):1-10.

65. Leitch EC, Walker AW, Duncan SH, Holtrop G, Flint HJ. Selective colonization of insoluble substrates by human faecal bacteria. Environ Microbiol. 2007;9(3):667-79.

66. Koropatkin NM, Cameron EA, Martens EC. How glycan metabolism shapes the human gut microbiota. Nat Rev Microbiol. 2012;10(5):323-35.

67. Turroni F, Peano C, Pass DA, Foroni E, Severgnini M, Claesson MJ, Kerr C, Hourihane J, Murray D, Fuligni F, Gueimonde M, Margolles A, De Bellis G, OToole PW, van Sinderen D, Marchesi JR, Ventura M. Diversity of bifidobacteria within the infant gut microbiota. PLoS One. 2012;7(5):e36957.

68. Adlerberth I, Wold AE. Establishment of the gut microbiota in Western infants. Acta Paediatr. 2009;98(2):229-38.

69. Ren PG, Zhou PJ. Salinibacillus aidingensis gen. nov., sp. nov. and Salinibacillus kushneri sp. nov., moderately halophilic bacteria isolated from a neutral saline lake in Xin-Jiang, China. Int J Syst Evol Microbiol. 2005;55(Pt 2):949-53.
70. Ludwig W, Strunk O, Westram R, Richter L, Meier H, Yadhukumar, Buchner A Lai T, Steppi S, Jobb G, Förster W, Brettske I, Gerber S, Ginhart AW, Gross O, Grumann S, Hermann S, Jost R, König A, Liss T, Lüssmann R, May M, Nonhoff B, Reichel B, Strehlow R, Stamatakis A, Stuckmann N, Vilbig A, Lenke $M$, Ludwig T, Bode A, Schleifer KH. ARB: a software environment for sequence data. Nucleic Acids Res. 2004;32(4):1363-71.

\section{Submit your next manuscript to BioMed Central and we will help you at every step:}

- We accept pre-submission inquiries

- Our selector tool helps you to find the most relevant journal

- We provide round the clock customer support

- Convenient online submission

- Thorough peer review

- Inclusion in PubMed and all major indexing services

- Maximum visibility for your research

Submit your manuscript at www.biomedcentral.com/submit
Biomed Central 